\title{
ON THE REDUCTION OF THE RESISTANCE IN THE CENTRAL ARTERIAL VESSEL
}

\author{
Kuralbay Navruzov ${ }^{1}$, Suxrob Rajabov ${ }^{2}$, Zoxid Shukurov ${ }^{3}$, Begjanov \\ Amirbek ${ }^{4}$, Babajanova Yulduz
}

1Professor, department of applied mathematics and mathematical physics, Urganch state University, Republic of Uzbekistan

${ }^{2}$ Teacher, department of applied mathematicsand mathematical physics, Samarkand State University, Republic of Uzbekistan

${ }^{3}$ Teacher, department of applied mathematics Samarkand Institute of Economics and Service, Republic of Uzbekistan

${ }^{4}$ Urganch state university, Republic of Uzbekistan

5Urganch state university, Republic of Uzbekistan

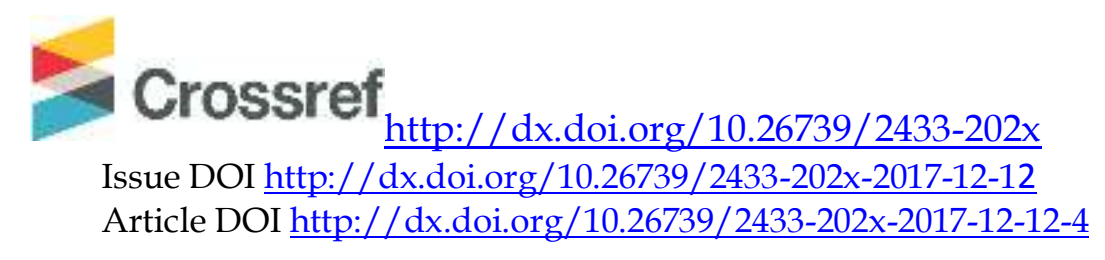

Abstract: The article deals with the stationary blood flow in vessels with permeable walls. To determine the hydraulic resistance in an arterial vessel, the blood is considered to be a Newtonian viscous liquid, and the flow is stationary. In solving the problem, formulas are obtained for determining the corresponding hydrodynamic parameters, such as velocity, fluid flow and pressure gradient. The hydraulic resistance is determined with the using of impedance method. For a stationary flow of hydraulic resistance in a permeable vessel, it depends significantly on the permeability coefficient, and with an increase in this coefficient it decreases

Keywords: Newton's liquids, continuity, oscillation parameter, pulsating melodeons 


\section{INTRODUCTION}

During reduction of heart from heart on walls of blood vessels pressure wave which is called a pulse wave extends. This wave in process of removal from heart gradually weakens and practically fades in capillaries. Speed of distribution of a pulse wave depends on many factors among which it is possible to note, for example, elastic and viscous properties of a wall of a vessel, the blood pressure, its density, viscosity, permeability of a wall of vessels, etc. In the majority of works [1-6] the main attention is paid on definition of distribution of a pulse wave of pressure taking into account elasticity of a wall of vessels, and its permeability isn't considered anywhere. However, the permeability of a wall significantly influences distribution of a pulse wave of pressure and its attenuation.

Branching of an arterial tree is modelled by introduction of "permeability" of a wall with volume outflows of cross speed on walls. Really, this speed strongly explosive function of coordinate, when using model of a permeable tube function always smoothes out a wall surface. Apparently, the idea of modeling of an artery in the form of a permeable tube has been for the first time stated in work [7] and developed further in [8]. The fullest research of such model contains in work [9] where the blood circulatory system of a dog is modelled. Unlike other works [2-6,11], for determination of hydraulic resistance in the central arterial vessel in works $[10,11]$ problem definition of the pulsing current of blood in the arterial course where outflow of blood is mathematically modelled in the form of a permeable wall of vessels is considered. It should be noted that except wave streams, it is expedient to study a stationary current of blood in a vessel with permeable walls. However, this case doesn't follow from the decision for wave streams a limit perekhokd at aspiration of frequency to zero therefore the stationary task should be considered in separate statement.

\section{Problem definition and methods of the decision}

At a stationary current of liquid take place of a ratio

$$
\frac{\partial v_{x}}{\partial t}=0, \frac{\partial v_{r}}{\partial t}=0, \frac{\partial u_{x}}{\partial t}=\frac{\partial u_{r}}{\partial t}=0
$$

\section{Health science}


Considering them, from [1-5] we receive the system of the equations.

$$
\left\{\begin{array}{l}
\frac{1}{\rho} \frac{\partial p}{\partial x}=v\left(\frac{\partial^{2} v_{x}}{\partial r^{2}}+\frac{1}{r} \frac{\partial v_{x}}{\partial r}+\frac{\partial^{2} v_{x}}{\partial x^{2}}\right) \\
\frac{1}{\rho} \frac{\partial p}{\partial r}=v\left(\frac{\partial^{2} v_{r}}{\partial r^{2}}+\frac{1}{r} \frac{\partial v_{r}}{\partial r}-\frac{1}{r^{2}} v_{r}+\frac{\partial^{2} v_{r}}{\partial x^{2}}\right) \\
\frac{\partial v_{r}}{\partial r}+\frac{1}{r} v_{r}+\frac{\partial v_{x}}{\partial x}=0
\end{array}\right.
$$

and boundary conditions

$$
\left\{\begin{array}{l}
v_{r}=\frac{R \gamma *}{\mu}\left(p-p_{c}\right), v_{x}=0, r=R \\
\frac{\partial v_{x}}{\partial r}=0, \quad v_{r}=0, \quad r=0 \\
p=p_{0}, \quad Q=Q_{0}, \quad x=0
\end{array}\right.
$$

Where $\rho$-blood density, $\mathrm{r}$ - radial, $x$-longitudinal coordinates, $v_{r}$ and $v_{x}$ - the radial and axial being speeds, $v$-kinematic viscosity of blood, $p$ - internally pressure, $p_{c}$ - pressure in the environment, $p_{0}, Q_{0}$ average values of pressure and expense, $\gamma *$ - permeability coefficient.

From system (1), having made some calculations, we will find the equation for pressure

$$
\frac{\partial^{2} p}{\partial r^{2}}+\frac{1}{r} \frac{\partial p}{\partial r}+\frac{\partial^{2} p}{\partial x^{2}}=0
$$

according to which in a stationary stream distribution of pressure submits to Laplace's equation. We look for the decision (3) in a look

$$
p=p_{1}(r) \exp \left(i \gamma_{n} x\right)+p_{2}(r) \exp \left(-i \gamma_{n} x\right)
$$

\section{Health science}


This technique follows from the common decision [3] provided that. In this case the complex argument will be transformed to valid, etc.

$$
p=p_{1}(r) \exp \left(\frac{\lambda}{R} x\right)+p_{2}(r) \exp \left(-\frac{\lambda}{R} x\right)
$$

here all functions valid. Therefore in a stationary task there is no need of the complex decision.

Substitution (5) in (3) leads to Bessley's equation.

$$
\frac{d^{2} p_{1,2}}{d r^{2}}+\frac{1}{r} \frac{d p_{1,2}}{d r}+\frac{\lambda^{2}}{R^{2}} p_{1,2}=0
$$

His fundamental decisions will be:

$$
J_{0}\left(\frac{\lambda}{R} r\right) \quad \text { and } \quad Y_{0}\left(\frac{\lambda}{R} r\right)
$$

In this case the common decision has an appearance

$$
p_{1,2}=c_{1,2} J_{0}\left(\frac{\lambda}{R} r\right)+d_{1,2} Y_{0}\left(\frac{\lambda}{R} r\right)
$$

at $\mathrm{r}=0$, pressure addresses in infinity therefore coefficients before function

$$
Y_{0}\left(\frac{\lambda}{R} r\right)
$$

have to equal to zero and in this case the decision (6) takes a form

$$
p_{1,2}=c_{1,2} J_{0}\left(\frac{\lambda}{R} r\right)
$$

or it is final, we will receive the decision

$$
p-p_{c}=J_{0}\left(\frac{\lambda}{R} r\right)\left[c_{1} \exp \left(\frac{\lambda}{R} x\right)+c_{2} \exp \left(-\frac{\lambda}{R} x\right)\right]
$$

\section{Health science}


where coefficients $\quad \mathrm{C}_{1}$ and $\mathrm{C}_{2}$ are defined from boundary conditions (2). Account (8) for the first equation of system (1) gives the equation

$$
\frac{\partial^{2} v_{x}}{\partial r^{2}}+\frac{1}{r} \frac{\partial v_{x}}{\partial r}+\frac{\partial^{2} v_{x}}{\partial x^{2}}=\frac{\lambda}{\mu R} J_{0}\left(\frac{\lambda}{R} r\right)\left[c_{1} \exp \left(\frac{\lambda}{R} x\right)-c_{2} \exp \left(-\frac{\lambda}{R} x\right)\right]
$$

which decision we look for in a look

$$
v_{x}=v_{1 x}(r)\left[c_{1} \exp \left(\frac{\lambda}{R} x\right)-c_{2} \exp \left(-\frac{\lambda}{R} x\right)\right]
$$

Then

$$
\frac{d^{2} v_{1 x}(r)}{d r^{2}}+\frac{1}{r} \frac{d v_{1 x}(r)}{d r}+\frac{\lambda^{2}}{R^{2}} v_{1 x}=\frac{\lambda}{\mu R} J_{0}\left(\frac{\lambda}{R} r\right)
$$

Equation (11) is solved under the condition that the fluid adheres to the wall of the tube,i.e.

$v_{x}=0 \quad$ at $r=R$ and limitations $v_{x}<\infty$ on the axis of the pipe.

Fundamental decisions (11) functions of Bessel нулево $г$ го about will be expressed so

$$
J_{0}\left(\frac{\lambda}{R} r\right) \quad \text { u } \quad Y_{0}\left(\frac{\lambda}{R} r\right)
$$

The equation (11) is non-uniform therefore we find his private reksheniye from a formula

$$
v^{*}{ }_{1 x}=\frac{\pi}{2} Y_{0}(x) \int x J_{0}(x) f(x) d x-\frac{\pi}{2} J_{0}(x) \int x Y_{0}(x) f(x) d x
$$

Where

$$
f(x)=\frac{\lambda}{\mu R} J_{0}(x), \quad x=\frac{\lambda}{R} r
$$

Sometimes, considering a ratio

\section{Health science}




$$
J_{0}(x) Y_{0}^{\prime}(x)-J_{0}^{\prime}(x) Y_{0}(x)=\frac{2}{\pi x}
$$

We get it

$$
v *_{1 x}=\frac{1}{2 \mu} r J_{1}\left(\frac{\lambda}{R} r\right)
$$

The use of the fundamental solution (12) and the limited velocity on the axis of the tube gives

$$
v_{1 x}=c_{1} J_{0}\left(\frac{\lambda}{R} r\right)+\frac{1}{2 \mu} r J_{1}\left(\frac{\lambda}{R} r\right)
$$

From condition $v_{x}=0$ at $\mathrm{r}=\mathrm{R}$, define

$$
\overline{c_{1}}=-\frac{1}{2 \mu} \frac{R J_{1}(\lambda)}{J_{0}(\lambda)}
$$

Substituting (17) into (16), from (10) we find

$$
v_{x}=\frac{R}{2 \mu}\left\{\frac{r}{R} J_{1}\left(\frac{\lambda}{R} r\right)-\frac{J_{1}(\lambda)}{J_{o}(\lambda)} J_{0}\left(\frac{\lambda}{R} r\right) \times\left\{c_{1} \exp \left(\frac{\lambda}{R} x\right)-c_{2} \exp \left(-\frac{\lambda}{R} x\right)\right\}\right.
$$

In the same way by means of the decision (18) and the second equation of system (1) we will receive a formula for distribution of cross speed:

$$
v_{r}=\frac{R}{2 \mu \lambda}\left\{\frac{\lambda r}{R} J_{0}\left(\frac{\lambda}{R} r\right)+\left[\frac{\lambda J_{1}(\lambda)}{J_{0}(\lambda)}-1\right] J_{1}\left(\frac{\lambda}{R} r\right)\right\} \times\left(\mathrm{c}_{1} \exp \left(\frac{\lambda}{\mathrm{R}} x\right)+\mathrm{c}_{2} \exp \left(-\frac{\lambda}{R} x\right)\right)
$$

Use of a condition of permeability of a wall (2) leads to the equation for determination of own value $\lambda$

$$
\gamma *=\frac{1}{2 \lambda}\left\{\lambda+\frac{J_{1}(\lambda)}{J_{0}(\lambda)}\left[\lambda \frac{J_{1}(\lambda)}{J_{0}(\lambda)}-1\right]\right\}
$$

\section{Health science}


If $j^{*} \ll I$ having spread out functions $J_{0}(\lambda), J_{1}(\lambda)$ in convergent series

$$
\left\{\begin{array}{l}
J_{0}(z)=1-\frac{\left(\frac{z}{2}\right)^{2}}{1}+\frac{\left(\frac{z}{2}\right)^{4}}{1^{2} 2^{2}}-\frac{\left(\frac{z}{2}\right)^{6}}{1^{2} 2^{2} 3^{2}}+\ldots \\
J_{1}(z)=\frac{z}{2}-\frac{\left(\frac{z}{2}\right)^{3}}{1^{2} 2}+\frac{\left(\frac{z}{2}\right)^{5}}{1^{2} 2^{2} 3}+\ldots
\end{array}\right.
$$

from (20) after some calculations we will find

$$
\lambda=4 \sqrt{\gamma *}
$$

Equation (20) is transcendental and has an infinite set of roots . Summation on own znachenikyam gives formulas for pressure, radial and axial velocities. $p-p_{c}=\sum_{n=1}^{\infty} J_{0}\left(\frac{\lambda_{n}}{R} r\right)\left[A_{n} \exp \left(\frac{\lambda_{n}}{R} x\right)+B_{n} \exp \left(-\frac{\lambda_{n}}{R} x\right)\right]$

\section{Health science}




$$
\begin{aligned}
& \left\{\begin{array}{l}
v_{x}=\sum_{n=1}^{\infty} \frac{R}{2 \mu}\left\{\frac{r}{R} J_{1}\left(\frac{\lambda_{n}}{R} r\right)-\frac{J_{1}\left(\lambda_{n}\right)}{J_{0}\left(\lambda_{n}\right)} J_{0}\left(\frac{\lambda_{n}}{R} r\right)\right\} \times\left\{A_{n} \exp \left(\frac{\lambda_{n}}{R} x\right)-B_{n} \exp \left(-\frac{\lambda_{n}}{R} x\right)\right\} \\
v_{r}=\sum_{n=1}^{\infty} \frac{R}{2 \mu \lambda_{n}}\left\{\frac{\lambda_{n} r}{R} J_{0}\left(\frac{\lambda_{n}}{R} r\right)+\left[\frac{\lambda_{n} J_{1}\left(\lambda_{n}\right)}{J_{0}\left(\lambda_{n}\right)}-1\right] J_{0}\left(\frac{\lambda_{n}}{R} r\right)\right\} \times \\
\times\left\{A_{n} \exp \left(\frac{\lambda_{n}}{R} x\right)+B_{n} \exp \left(-\frac{\lambda_{n}}{R} x\right)\right\} \\
\left\{\begin{array}{l}
\frac{\mu \lambda_{n}{ }^{2} Q_{0}}{\pi R^{3}} \\
A_{n}=\frac{\lambda_{n}\left(\bar{p}-p_{c}\right)}{4 J_{1}(\lambda)}-\frac{\left.2\left(\lambda_{n} J_{0}\left(\lambda_{n}\right)+J_{1}\left(\lambda_{n}\right)\left[\frac{\lambda_{n} J_{1}\left(\lambda_{n}\right)}{J_{0}\left(\lambda_{n}\right)}-1\right]\right)\right)}{2\left(\left(\lambda_{n} J_{0}\left(\lambda_{n}\right)+J_{1}\left(\lambda_{n}\right)\left[\frac{\lambda_{n} J_{1}\left(\lambda_{n}\right)}{J_{0}\left(\lambda_{n}\right)}-1\right]\right)\right)}
\end{array}\right.
\end{array}\right.
\end{aligned}
$$

\section{RESULTS AND THEIR DISCUSSION}

Formulas (23) allow to investigate characteristics of vnuktrenny structure of a current of viscous blood in a pipe with pronitsayekmy walls: hydrodynamic resistance on a permeable wall, a liquid consumption on length of a pipe and some other the gidrodinakmicheskikh of parameters. Using (23), we find distribution of pressure difference, a consumption of blood and tangent tension on a wall:

$$
\begin{aligned}
& \quad-\frac{\partial p}{\partial x}=-\sum_{n=1}^{\infty} J_{0}\left(\frac{\lambda_{n}}{R} r\right)\left[A_{n} \frac{\lambda_{n}}{R} \exp \left(\frac{\lambda_{n}}{R} x\right)-\frac{\lambda_{n}}{R} B_{n} \exp \left(-\frac{\lambda_{n}}{R} x\right)\right] \\
& Q=2 \pi \int_{0}^{R} r v_{x}(r, x) d r= \\
& =\frac{\pi R^{3}}{\mu} \sum_{n=1}^{\infty}\left\{\frac{1}{\lambda_{n}^{2}} J_{0}\left(\lambda_{n}\right) \times\left\{\lambda+\frac{J_{1}\left(\lambda_{n}\right)}{J_{0}\left(\lambda_{n}\right)}\left[\frac{J_{1}\left(\lambda_{n}\right) \lambda_{n}}{J_{0}\left(\lambda_{n}\right)}-1\right]\right\}\left(B_{n} \exp \left(-\frac{\lambda_{n}}{R} x\right)-A_{n} \exp \left(\frac{\lambda_{n}}{R} x\right)\right)\right\} \\
& \tau_{c p}=\frac{1}{L} \int_{0}^{L} \mu\left(\frac{\partial v_{x}}{\partial r}+\frac{\partial v_{r}}{\partial x}\right) \mid r=R \\
& =\sum_{n=1}^{\infty}\left\{\frac{R}{L \lambda_{n}} J_{0}\left(\lambda_{n}\right)\left(2 \lambda_{n} \gamma *+\frac{J_{1}\left(\lambda_{n}\right)}{J_{0}\left(\lambda_{n}\right)}\right)\left[\left(A_{n} \exp \left(\frac{\lambda_{n}}{R} L\right)+B_{n} \exp \left(-\frac{\lambda_{n}}{R} L\right)-\left(A_{n}+B_{n}\right)\right)\right]\right\}
\end{aligned}
$$

\section{Health science}


By means of formulas (25) and (26) we will define pressure difference relation per unit length to a blood consumption:

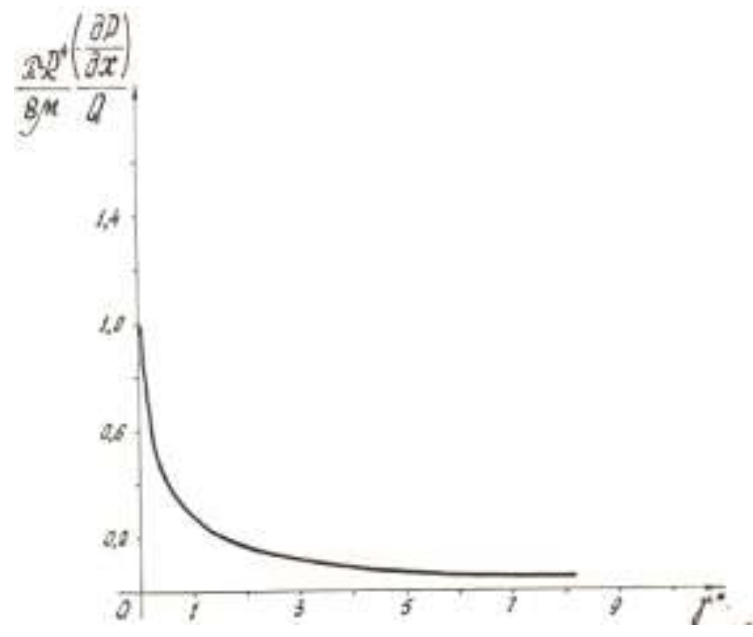

Fig. 1

Change of full resistance of blood in the arterial course depending on permeability of a wall 


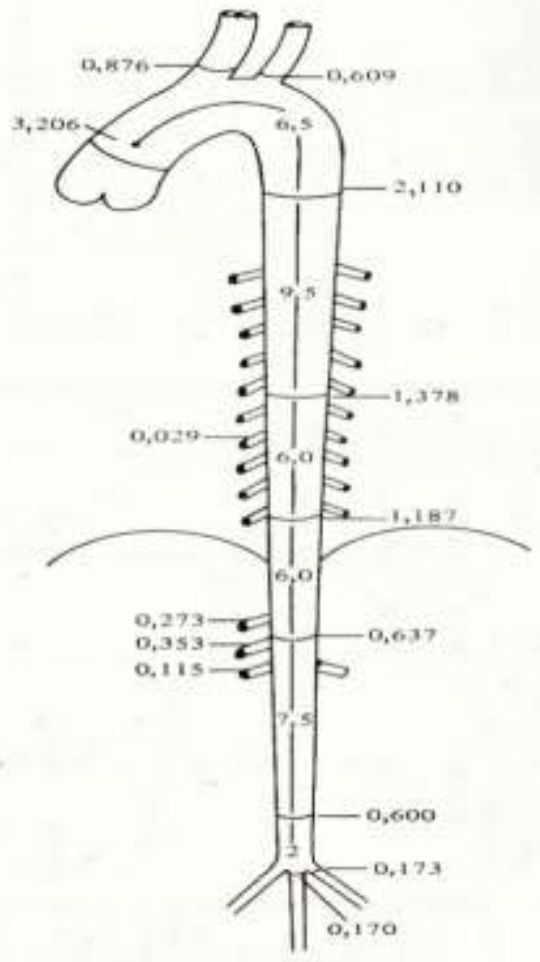

The area of the internal cross section ( $\mathrm{cm} 2)$ of an aorta and artery in various points of its main branches for a dog [2].

$\frac{\left(-\frac{\partial \bar{p}}{\partial x}\right)}{Q}=\frac{8 \mu}{\pi R^{4}} \sum_{n=1}^{\infty} \frac{\lambda_{n}}{8 \gamma *} \frac{J_{1}\left(\lambda_{n}\right)}{J_{0}\left(\lambda_{n}\right)}$

or, in a dimensionless look,

$\frac{\pi R^{4}}{8 \mu} \frac{\left(-\frac{\partial \bar{p}}{\partial x}\right)}{Q}=\sum_{n=1}^{\infty} \frac{\lambda_{n}}{8 \gamma *} \frac{J_{1}\left(\lambda_{n}\right)}{J_{0}\left(\lambda_{n}\right)}$ 
The relation is called an effective impedance, generally it is expressed by function of complex variable. Therefore, his valid part determines the hydraulic resistance of a stream. From fig. 1 it is visible that the effective impedance at a stationary stream in a permeable pipe significantly depends on permeability coefficient: with increase in this coefficient he decreases. At mathematical modeling of a current of blood in the arterial course where outflow of blood from the main trunk is considered in the form of a permeable wall of a vessel. For the quantitative analysis we will provide data from work [2] for a dog. By means of fig. 2. we will calculate the sum of the cross areas of a side branch, she makes about $3.6 \mathrm{~cm} 2$, and the superficial area of the main arterial trunk is $240 \mathrm{~cm} 2$. The coefficient of permeability of a wall is defined by the relation of the total cross areas of a side branch to the superficial area of the main trunk. In this case she is equal to 0.015 . From fig. 1 it is visible that in an arterial vessel hydraulic resistance decreases due to permeability of a wall when she makes value 0.015 , corresponds to reduction by $9-10$ percent. 


\section{REFERENCE}

1. Navruzov K., Hakberdiyev Zh. B. Dynamics of non-Newtonian liquids. Tashkent, 2000. 246 pages.

2. T. Gidrodinamik's loops of large blood vessels M. Mir. 1983. 400 pages.

3. Fayzullayev D. F., Navruzov of K. Gidrodinamik of the pulsing streams. Tashkent, 1986. 192 pages.

4. Navruzov K. Hydrodynamics of the pulsing currents in pipelines.

Tashkent, 1986. 112 pages.

5. Navruzov K.N. Biomechanics of large blood vessels. Tashkent, "Fan ва technology", 2011, 144 pages.

6. Navruzov K.N., Abdukarimov F. B, Huzhatov N.Zh. To the theory of hydraulic resistance in a pulse current of blood in vessels with mobile walls/ /"Ilm of a sarchashmalari", UrSU, 2014, No. 4, page 16-19.

7. Streeter, V.L., Keitzer, W.F., Bohr F.F. Pulsatile pressure and flow through distensible vessels. Circ.Res. 1963, v.13, 3-20

8. Skalak, R., Stathis, T. A porous tapered elashis tube model of a vascular. Bed. In. Biomechanics symposium ed. Y.C.Fung.1966, ASME. New.York. 9.Anliker, M., Rockwell, R.L., Ogden, E. Nonlinear analysis of flow pulses and shock waves in arteries. I.\&.II. Angew.Math. Phys.1971, v.22, 217-246 10. Navruzov K.N., Abdukarimov F.B. Hydrodynamics of the pulsing currents of blood. Germany, "Lap-Lambert", 2015, 209 pages.

11. Navruzov K.N. An impedance method of determination of hydraulic resistance in arterial vessels (problem definition)//"Ilm of a sarchashmalara", URSU, 2016, No. 7 of page $20-23$ 